\title{
CASE REPORT \\ Cryptococcal laryngitis: An uncommon presentation of a common pathogen
}

\author{
Y Atiya, MB BCh, MMed (ORL), FCORL (SA); S D Masege, MB ChB, FCORL (SA) \\ Division of Otorhinolaryngology - Head Neck Surgery, Department of Neurosciences, Faculty of Health Sciences, \\ Chris Hani Baragwanath Hospital and University of the Witwatersrand, Johannesburg, South Africa
}

Corresponding author: Y Atiya (yatiya@gmail.com)

Cryptococcus neoformans is a ubiquitous encapsulated yeast found worldwide, especially in areas with pigeons. The fungus thrives in pigeon droppings and is responsible for primary pulmonary infection, but may disseminate and cause infection of the central nervous system, skin and bone. Most cases are reported in immunocompromised hosts, most commonly those infected with HIV. However, infection has been reported in immunocompetent hosts. Primary infection of the larynx is uncommon, and to date only 12 cases have been reported. We present the first South African report of a young woman with HIV who presented with hoarseness of uncertain aetiology, which was later confirmed to be cryptococcal laryngitis.

S Afr Med J 2015;105(10):875. DOI:10.7196/SAMJnew.8779

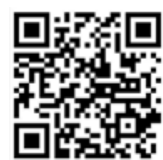

Cryptococcus neoformans is a ubiquitous encapsulated yeast found worldwide, especially in areas with pigeons. The fungus thrives in pigeon droppings and is responsible for primary pulmonary infection, but may disseminate and cause infection of the centra nervous system, skin and bone. ${ }^{[1]}$ Most cases are reported in immunocompromised hosts, most commonly those infected with HIV. However, infection has been reported in immunocompetent hosts. ${ }^{[1,2]}$ Primary infection of the larynx is uncommon, and to date only 12 cases have been reported. ${ }^{[3]}$ We present the first South African report of a young woman with HIV who presented with hoarseness of uncertain aetiology, which was later confirmed to be cryptococcal laryngitis.

\section{Case report}

A 38-year-old woman presented to the outpatient department complaining of

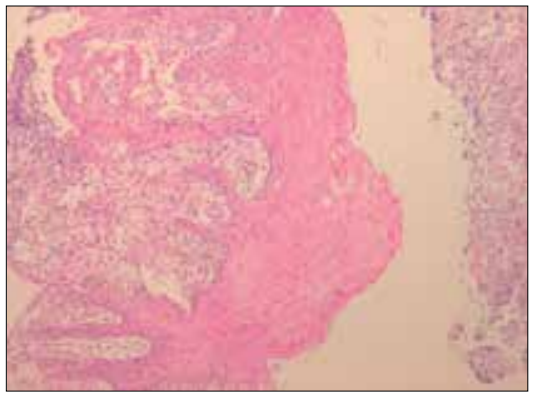

Fig. 1. Low-power haematoxylin-eosin stain showing fungal spores in the submucosa. Note the squamous hyperplasia. hoarseness of 7 months' duration, which had progressively worsened. She had no difficulty breathing, had never smoked, and was unemployed. There was no history of intubation or a preceding upper-respiratory tract infection. She had been diagnosed with HIV infection some months before, with a CD4 count of 220 cells $/ \mu \mathrm{L}$, and was not on antiretroviral medication. She had never had tuberculosis and had previously been well.

Examination revealed a relatively healthy woman with oral thrush and a rash of the lower extremities that was initially thought to be Kaposi sarcoma. She had no other stigmata of retroviral disease. Her otolaryngological examination was unremarkable, except that flexible nasopharyngoscopy revealed thickening of both true vocal folds, which was more pronounced on the right, with normal mobility of the folds. The lesions did not appear obviously malignant, and there was no associated cervical lymphadenopathy.

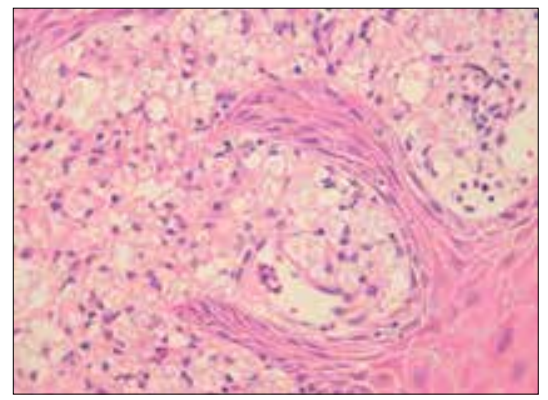

Fig. 2. High-power haematoxylin-eosin stain. The fungal spores are evident as numerous small red entities.
Workup included a chest radiograph, which was normal, and sputum for microscopy as well as examination for acid-fast bacilli (AFB). The microscopy was strongly positive for C. neoformans; no AFB were detected. An abdominal ultrasound was also normal.

She was taken to theatre for a direct laryngoscopy and biopsy of the vocal fold lesions. The clinical findings were confirmed in theatre, and no further abnormalities were detected.

Histological examination of the tissue biopsies showed multiple fungal spores, with thick capsules, in the submucosa (Figs 1 - 3). There was no evidence of granulomatous disease or malignancy. The epithelium was hypertrophied.

The patient was treated with intravenous fluconazole for 14 days, then continued on oral doses for a further month, to be reviewed as an outpatient. On discharge, her voice had improved. Unfortunately, she was lost to follow-up.

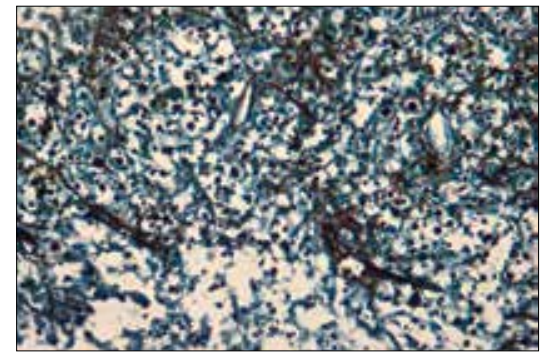

Fig. 3. Grocott stain. The fungus appears black, on a green background. In this case, it is obscured owing to the high load of organisms, and the entire slide appears black. 


\section{Discussion}

Before the AIDS era, cryptococcal infection was uncommon, and usually occurred in patients with other immunosuppressive conditions such as haematological malignancy, longterm steroid use and diabetes. ${ }^{[3,4]}$ Currently, it is one of the most common life-threatening infections in patients with AIDS. ${ }^{[2]}$ The most common infection is pulmonary, which is usually self-limiting. Central nervous system involvement results in high morbidity and mortality; mortality rates of up to $30 \%$ have been reported. ${ }^{[2]}$ Laryngeal involvement is extremely uncommon and has only been reported in 12 other patients. $^{[3]}$ Features of these cases are outlined in Table 1. Hypotheses to explain laryngeal involvement include haematogenous spread from a primary focus, such as the lung, or direct implantation by inhalation. ${ }^{[1]}$

The most common complaint is hoarseness, usually of several months' duration. The appearance of the vocal folds varies, from erythematous, oedematous to leukoplakic and cystic.

Direct laryngoscopy and biopsy are essential to exclude other more common causes of vocal fold abnormalities, including malignancy and respiratory papillomatosis. ${ }^{[3]}$ Grossly, cryptococcal laryngitis has been reported to simulate laryngeal carcinoma. ${ }^{[1]}$ Therefore, the suspicion of fungal infection should be communicated to the pathologist so that appropriate staining can be done, or the diagnosis could be missed, as has been reported in a case. ${ }^{[3]}$ Other fungal infections may simulate carcinoma and even Kaposi sarcoma. ${ }^{[1]}$

There are no evidence-based guidelines for the treatment of laryngeal cryptococcosis. ${ }^{[3]}$ Advice from infectious disease specialists is therefore prudent. Assessment should include exclusion of HIV and other immunosuppressive conditions, and a search for disseminated disease.

Treatment options include amphotericin B and fluconazole. The former is only available as an intravenous formulation and is nephrotoxic. Fluconazole, however, has excellent oral

Table 1. Characteristics of previously reported patients with cryptococcal laryngitis

\begin{tabular}{ll}
\hline Cases & 12 \\
Age at presentation (years) & Mean 58 (31 - 87) \\
Male:female & $3: 1$ \\
Duration of hoarseness (months) & Mean $4(1-12)$ \\
Comorbidities/cofactors & Local exposure \\
& Inhaled steroids \\
& Tobacco smoking \\
& Chicken manure \\
& Tuberculosis \\
& Systemic factors \\
& Oral steroids \\
& Infection: HIV, hepatitis C \\
Findings on direct laryngoscopy (patients, $n$ ) & Diabetes mellitus \\
& Previous cryptococcal pneumonia \\
& Leukoplakia (4) \\
& Oedema (3) \\
Histopathology (patients, $n$ ) & Vocal fold erythema (3) \\
Treatment (patients, $n$ ) & Pseudo-epitheliomatous hyperplasia (5) \\
Duration of antifungal treatment (weeks) & Granulomatous inflammation (9) \\
Outcome (resolution:recurrence:unknown) & Squamous cell hyperplasia (1) \\
Modified from Gordon DH, et al.. ${ }^{(3)}$ with permission. & Submucosal cyst (1) \\
& Antifungal therapy (10) \\
& Endoscopic polypectomy (2) \\
& Mean 13.5 (4 - 40) \\
& \\
& \\
&
\end{tabular}

absorption and was the most commonly used agent in previous cases. ${ }^{[3]}$ The average duration of treatment was 13.5 (range 4 - 40) weeks.

\section{Conclusion}

Although uncommon, otolaryngologists should remain vigilant and consider the possibility of crytococcal infection of the larynx, particularly in immunocompromised hosts and patients presenting with unusual or protracted symptoms. Prompt consultation with an infectious disease specialist and treatment can prevent significant morbidity and/or mortality.
Acknowledgements. We acknowledge the assistance of Dr Gillian Davies Anamourlis of the Department of Anatomical Pathology for her assistance in preparing the high-resolution microscope images.

\section{References}

1. McGregor DK, Citron D, Shahab I. Cryptococcal infection of the larynx simulating laryngeal carcinoma. South Med J 2003;96(1):7477. [http://dx.doi.org/10.1097/01.SMJ.0000047976.06958.D6]

2. Chongkolwatana C, Suwanagool S, Chongvisal S. Primary cryptococcal infection of the larynx in a patient with AIDS: A case report. J Med Assoc Thai 1998;81(6):462-467.

3. Gordon DH, Stow NW, Yapa M, et al. Laryngeal cryptococcosis Clinical presentation and treatment of a rare cause of hoarseness. Otolaryngol Head Neck Surg 2010;142(3 Suppl):S7-S9. [http:// dx.doi.org/10.1016/j.otohns.2009.08.030]

4. Nadrous HF, Lewis JE, Ryu JH, Sabri AN. Cryptococcal laryngitis: Case report and review of the literature. Ann Otol laryngitis. Case 